\title{
Estudio cinético comparativo del proceso de digestión anaerobia del alpechín en los intervalos mesofílico y termofílico de temperatura.
}

\author{
Por R. Borja Padilla (1), A. Martín Martín (2), M. M. Durán Barrantes (1) y R. Maestro Durán (1)
}

(1) Instituto de la Grasa y sus Derivados (C.S.I.C.). Avda. Padre García Tejero, n.ํ 4. 41012 - SEVILLA. (2) Dpto. de Ingeniería Quimica. Facultad de Ciencias. Universidad de Córdoba.

Avda. San Alberto Magno, s/n. 14004 - CORDOBA.

\section{RESUMEN}

Estudio cinético comparativo del proceso de digestión anaerobia del alpechín en los intervalos mesofílico y termofílico de temperatura.

Se ha efectuado un estudio cinético del proceso de digestión anaerobia del alpechin en los intervalos mesofílico (de $30^{\circ} \mathrm{C}$ a $40^{\circ} \mathrm{C}$ ) y termofilico (de $50^{\circ} \mathrm{C}$ a $60^{\circ} \mathrm{C}$ ) de temperatura, utilizando dos reactores de lecho fluidizado con biomasa suspendida.

Se aplica el modelo de utilización de sustrato propuesto por ChenHashimoto, obteniéndose los valores de la velocidad máxima de crecimiento específico de microorganismos, $\mu_{\max }$, y de la constante cinética del proceso, $\mathrm{K}$, para cada uno de los casos estudiados.

Se obtiene un aumento de los parámetros cinéticos $\mu_{\text {. }}$ y $\mathrm{K}$, del $55 \%$ y $34 \%$ respectivamente, en el proceso de digestion anaerobia termofilico respecto al mesofilico.

El valor máximo en la velocidad volumétrica de utilización de sustrato se alcanza para un tiempo de retención hidráulico de 7,7 y 11,6 dias en los procesos termofilico y mesofilico, respectivamente.

PALABRAS-CLAVE: Alpechín - Cinética - Depuración anaerobia Proceso mesofílico - Proceso termofílico.

\section{SUMMARY}

Kinetic study of anaerobic digestion process of olive mill wastewater in the mesophilic and thermophilic ranges of temperature.

A kinetic study of anaerobic digestion of olive mill wastewater in the mesophilic (from $30^{\circ} \mathrm{C}$ to $40^{\circ} \mathrm{C}$ ) and thermophilic (from $50^{\circ} \mathrm{C}$ to $60^{\circ} \mathrm{C}$ ) ranges of temperature has been carried out. Two reactors containing suspended biomass were used

The Chen-Hashimoto substrate utilization kinetic model has been applied, obtaining the values of the maximum specific growth rate of microorganisms, $\mu_{\max }$, and the kinetic constant, $\mathrm{K}$, for each case studied

The obtained results showed an increase of $55 \%$ and $34 \%$ for $\mu_{\max }$ and $\mathrm{K}$, respectively, of the thermophilic anaerobic digestion process in relation to the mesophilic process.

The substrate utilization maximum rate value was reached for the hydraulic retention times of 7,7 and 11,6 days. for the thermophilic and mesophilic processes, respectively.

KEY-WORDS: Anaerobic digestion - Kinetics - Mesophilic process Olive mill wastewater - Thermophilic process.

\section{INTRODUCCION}

El proceso de digestión anaerobia puede desarrollarse fundamentalmente en tres intervalos de temperatura: psicrofílico $\left(15-20^{\circ} \mathrm{C}\right)$, mesofílico $\left(30-40^{\circ} \mathrm{C}\right)$ y termofílico $\left(50-60^{\circ} \mathrm{C}\right)$, siendo los dos últimos los procesos más utilizados para el tratamiento de residuos de alta carga orgánica. En la bibliografía se detallan diversos estudios que comparan el rendimiento de eliminación de materia orgánica en procesos de depuración anaerobia de distintos residuos de gran poder contaminante, en los intervalos mesofílico y termofílico de temperatura (Lema et al., 1988; Marchaim, 1988; Sales et al., 1988; Demharter and Pfeiffer, 1988; Sriprasertsak et al., 1985; Vicent et al., 1985; Hashimoto, 1983; Dhavises et al., 1985 y Kandler, 1983).

En líneas generales la digestión anaerobia en el intervalo termofílico proporciona una serie de ventajas en relación con la llevada a cabo en fase mesofílica, como son (Chen, 1983 y Buhr, 1977):

- Las velocidades de reacción bioquímicas suelen ser mayores al aumentar la temperatura, alcanzándose altas velocidades metabólicas, al intervenir enzimas físicas y químicamente más estables.

- Se favorecen las propiedades físicas y químicas del agua en relación al proceso de depuración, produciéndose un aumento de la difusión, ionización y solubilidad de la mayoría de los compuestos orgánicos e inorgánicos, así como una disminución de su viscosidad, tensión superficial y constante dieléctrica.

- Presenta una menor conversión de sustrato en material celular y un mayor rendimiento de producción de metano.

El objetivo de este trabajo es obtener las constantes cinéticas que rigen el proceso de digestión anaerobia del alpechín, en los intervalos mesofílico y termofílico, comparando la velocidad volumétrica de utilización de sustrato, el porcentaje de eliminación de materia orgánica y el coeficiente de producción de metano en ambos casos.

\section{PARTE EXPERIMENTAL}

\subsection{Equipo experimental}

Está constituido por dos unidades de digestión anaerobia de un litro de volumen, provistas de agitación mag- 
nética, que se encuentran introducidas en cámaras termostatizadas a las temperaturas de $35^{\circ} \mathrm{C}$ y $55^{\circ} \mathrm{C}$, respectivamente (Borja et al., 1991). El biogás producido se pasa a través de una disolución de $\mathrm{NaOH}$ para eliminar el $\mathrm{CO}_{2}$ y se determina el volumen de metano producido en función del tiempo.

\subsection{Inóculo}

Como inóculo para el arranque de los digestores se utilizan lodos procedentes de una balsa de vertido de alpechín debidamente diluidos y neutralizados, cuya composición está recogida en el mencionado trabajo.

\subsection{Alpechín utilizado}

Las características del alpechín utilizado en esta serie de experimentos se resumen en la tabla I.

Tabla 1

Características del alpechín utilizado

\begin{tabular}{lc}
\hline $\mathrm{pH}$ & 5,2 \\
DQO & $52,5 \mathrm{~g} / \mathrm{l}$ \\
Sólidos totales & $45,0 \mathrm{~g} / \mathrm{l}$ \\
Sólidos minerales & $11,0 \mathrm{~g} / \mathrm{l}$ \\
Sólidos volátiles & $34,5 \mathrm{~g} / \mathrm{l}$ \\
Sólidos en suspensión totales & $13,8 \mathrm{~g} / \mathrm{l}$ \\
Sólidos en suspensión minerales & $3,8 \mathrm{~g} / \mathrm{l}$ \\
Sólidos en suspensión volátiles & $10,0 \mathrm{~g} / \mathrm{l}$ \\
Acidez volátil (ac. acético) & $0,15 \mathrm{~g} / 1$ \\
Alcalinidad & $0,60 \mathrm{~g} / 1$ \\
Nitrógeno $\mathrm{NH}_{4}^{+}$ & $0,09 \mathrm{~g} / \mathrm{l}$ \\
Fenoles totales (ac. cafeico) & $750 \mathrm{mg} / \mathrm{l}$ \\
Ortodifenoles (ac. cafeico) & $95 \mathrm{mg} / 1$ \\
\hline
\end{tabular}

\subsection{Procedimiento experimental}

\section{Etapa preliminar}

A las dos unidades de digestión anaerobia se adicionan inicialmente $750 \mathrm{ml}$ de agua destilada y $250 \mathrm{ml}$ del inóculo ya mencionado. Previamente a la realización de los experimentos se someten a un período de aclimatación durante un tiempo de dos meses, añadiéndose cargas volumétricas progresivas de alpechín en los dos reactores que se encuentran a las temperaturas de $35^{\circ} \mathrm{C}$ y $55^{\circ} \mathrm{C}$, respectivamente.

Para poder aplicar modelos cinéticos de utilización de sustrato (Chen y Hashimoto, 1980) es necesario conocer la cantidad de materia orgánica no biodegradable que se añade en la alimentación, puesto que se ha encontrado que algunos compuestos presentes en este residuo, fundamentalmente los de naturaleza fenólica, resultan difícilmente biodegradables por los microorganismos en condiciones internas de los digestores anaerobios (Sorlini et al., 1986; Borja et al., 1992). Por ello, previamente al estudio cinético en régimen continuo, se realiza un ensayo en régimen discontinuo, introduciendo en ambos digestores una carga volumétrica de $50 \mathrm{ml}$ del alpechín utilizado, manteniéndose el experimento hasta que la cantidad de metano producido fue inapreciable (12 días) y la materia orgánica residual en los efluentes se mantuvo constante, continuándose aún durante una semana más. Al final de este período se determinó la DQO de los efluentes, cuyo valor fue de $2,9 \mathrm{~g} / \mathrm{l}$ (rango mesofílico) y $2,0 \mathrm{~g} / \mathrm{l}$ (rango termofílico). Esta misma operación se repitió con cargas volumétricas de $75 \mathrm{ml}$ y $100 \mathrm{ml}$ obteniéndose idéntico resultado, de manera que los mencionados valores se consideraron representativos de la cantidad de materia orgánica no biodegradable.

\section{Desarrollo de los experimentos}

Tras esta etapa preliminar se realizan paralelamente dos series de experimentos en régimen continuo a las temperaturas de $35^{\circ} \mathrm{C}$ y $55^{\circ} \mathrm{C}$, respectivamente. En cada una de ellas se trabaja en régimen continuo con tiempos de retención hidráulicos de $40,35,30,25,20,15$ y 10 días. Cada experimento se mantuvo durante 20 días para asegurar la consecución de las condiciones de estado estacionario en los digestores.

Para cada tiempo de retención se determina, tras alcanzarse estas condiciones, el volumen de metano producido, asi como la DQO, acidez volátil y $\mathrm{pH}$ de los efluentes correspondientes a cada digestor. Todos los experimentos se realizan por duplicado.

\subsection{Técnicas analíticas}

Los análisis de los parámetros de caracterización de los distintos efluentes residuales se efectúan de acuerdo con "Standard Methods for the Examination of Water and Wastewater" (American Public Health Association, 1985).

\section{RESULTADOS}

En la tabla II se recogen los valores de la DQO de los efluentes obtenidos para los distintos caudales de alimentación ensayados, correspondientes a los tiempos de retención ya mencionados, así como el volumen de metano producido diariamente, en los digestores que operan en los rangos mesofílico y termofílico.

Tabla II

Valores de la DQO $(\mathrm{g} / \mathrm{l})$ de los efluentes y del volumen de metano ( $\mathrm{ml} / \mathrm{d}$ ía) en función del tiempo de retención hidráulico (dias).

\begin{tabular}{crrrrr}
\hline & \multicolumn{2}{c}{ MESOFILICO } & & \multicolumn{2}{c}{ TERMOFILICO } \\
\cline { 3 - 5 } \cline { 5 - 6 } Tiempo retención & DQO & $\begin{array}{c}\text { Vol. } \\
\text { metano }\end{array}$ & & DQO & $\begin{array}{c}\text { Vol. } \\
\text { metano }\end{array}$ \\
\hline 40 & 3,95 & 355 & 2,85 & 430 \\
35 & 4,20 & 405 & 3,00 & 490 \\
30 & 4,45 & 475 & & 3,20 & 580 \\
25 & 4,95 & 565 & & 3,50 & 680 \\
20 & 5,80 & 710 & & 4,05 & 830 \\
15 & 8,05 & 790 & & 5,05 & 1.050 \\
10 & 24,15 & 650 & 8,20 & 1.460 \\
\hline
\end{tabular}




\section{DISCUSION DE LOS RESULTADOS}

\subsection{Modelo cinético de utilización de sustrato de Chen-Hashimoto}

El diseño de equipos requiere la utilización de ecuaciones matemáticas que reflejen lo más fielmente posible el comportamiento del sistema bajo diferentes condiciones de operación. En el caso de procesos microbiológicos, las ecuaciones de diseño de los diferentes equipos utilizados se han basado, tradicionalmente, en el modelo de crecimiento de microorganismos propuesto por Monod (1949).

Sin embargo, diferentes autores (Pfeffer, 1984 y Benefield y Randall, 1987) han observado que este modelo no es capaz de predecir el comportamiento de determinados procesos, debido principalmente a que no considera la influencia que ejerce la concentración de sustrato presente en la alimentación sobre la concentración del efluente. Debido a ello, en los últimos años, se han propuesto numerosos modelos de crecimiento de microorganismos, entre los que destaca el propuesto por Contois, basado en la ecuación:

$$
\mu=\mu_{\max } \mathrm{S} /(B M+S)
$$

donde:

$\mu$ : velocidad específica de crecimiento de microorganismos.

$\mu_{\max }$ : velocidad máxima de crecimiento específico de microorganismos.

M: concentración de masa celular.

B: parámetro cinético adimensional.

S: concentración de materia orgánica biodegradable.

Este modelo tiene como principal inconveniente para su aplicación el que en los procesos anaerobios es muy difícil medir con precisión el valor de M. Por ello, basándose en él, Chen y Hashimoto desarrollaron un modelo de utilización de sustrato, en el que obvian dicho problema mediante la inclusión de un nuevo parámetro cinético adimensional $\mathrm{K}$, que representa un índice de inhibición del proceso:

$$
K=B Y
$$

donde $Y$ es el coeficiente de rendimiento celular, que en estado estacionario viene dado por:

$$
Y=M /(\text { So-S) }
$$

siendo So la concentración de materia orgánica biodegradable presente en la alimentación.

Estas modificaciones junto con los correspondientes balances de materia (aplicados al sustrato y a los microorganismos) dan lugar a la ecuación fundamental del modelo (Chen-Hashimoto, 1980):

$$
\theta=1 / \mu_{\max }+K(\text { So-S }) / \mu_{\max } S
$$

donde $\theta$ es el tiempo de retención hidráulico.
Según esta ecuación, la representación de $\theta$ frente a (So-S)/S debe dar lugar a una línea recta de ordenada en el origen $1 / \mu_{\max }$ y pendiente $\mathrm{K} / \mu_{\max }$, lo que permite el cálculo de los parámetros cinéticos $\mathrm{K}$ y $\mu_{\max }$.

\subsection{Aplicación del modelo cinético a los resulta- dos experimentales}

En la figura 1 se representan los valores del tiempo de retención hidráulico frente al cociente de concentraciones de sustrato biodegradable (So-S)/S, para los experimentos realizados en los rangos mesofílico y termofílico de temperatura. Los valores de So y $S$ se obtienen a partir de los datos de la DQO del alpechín utilizado (tabla 1) y de los efluentes (tabla II), teniendo en cuenta que la concentración de sustrato no biodegradable, expresado en DQO, es 2,9 y $2,0 \mathrm{~g} / \mathrm{l}$ en los rangos mesofílico y termofilico, respectivamente.

-Tiempo de retención hidráulico (días)
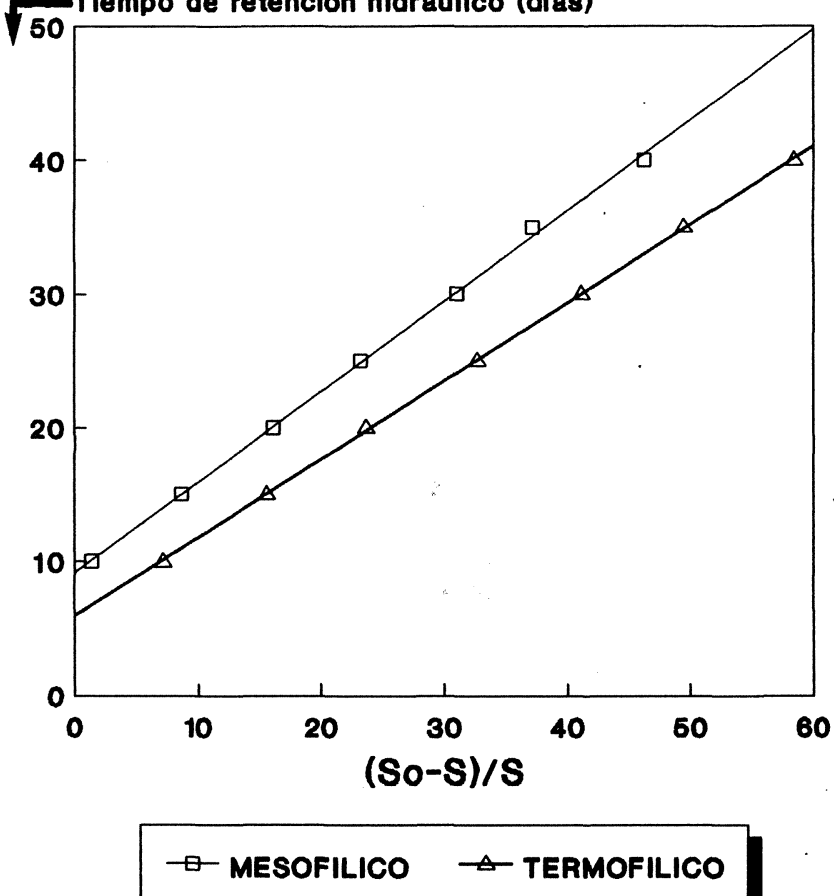

Figura 1

Determinación de los parámetros cinéticos $\mu_{\max }$ y $\mathrm{K}$ del modelo de Chen-Hashimoto, en los dos casos estudiados.

Las parejas de valores tiempo de retención, (So-S)/S, se ajustan a una linea recta, utilizando el método de mínimos cuadrados en los dos casos estudiados, de manera que según la ecuación (4) se pueden obtener los valores de los parámetros cinéticos $\mathrm{K}$ y $\mu_{\max }$ a partir de la pendiente y ordenada en el origen de ambas rectas. Los coeficientes de correlación obtenidos son bastante elevados en los dos casos, presentando un valor medio de 0,9996 . 
En la tabla III se resumen los valores de $\mu_{\max }$ y $\mathrm{K}$ con un intervalo de confianza del $95 \%$ para los dos rangos de temperatura estudiados. Por otra parte, los valores del tiempo de retención hidráulico mínimo, $\theta_{\min }$, se obtienen a través del inverso de $\mu_{\max }$ y son: 9,2 y 5,9 días para los procesos mesofílico y termofílico respectivamente. Los valores de los parámetros cinéticos obtenidos muestran que en el proceso termofílico la velocidad máxima de crecimiento específica y la constante cinética del proceso son un $55 \%$ y un $34 \%$ superiores, respectivamente, a los del proceso mesofílico, lo que da idea de la mayor velocidad con la que se producen las transformaciones celulares en el intervalo de $50-60^{\circ} \mathrm{C}$ de temperatura; ello supone la posibilidad de trabajar con tiempos de retención inferiores conservando una capacidad depurativa similar. En concreto, el tiempo mínimo de retención para el sistema es inferior en el caso del proceso anaerobio termofilico, dado que dicho parámetro es el inverso de la velocidad máxima de crecimiento específica.

Tabla III

Valores de $\mu_{\max }$ (dias $^{-1}$ ) y K (adimensional) con sus límites de confianza $(P<0,05)$ en los dos rangos de temperatura estudiados

\begin{tabular}{lll}
\hline & Mesofílico & Termofílico \\
\hline$\mu_{\max }$ & $0,109 \pm 0,007$ & $0,169 \pm 0,008$ \\
$\mathrm{~K}$ & $0,074 \pm 0,002$ & $0,099 \pm 0,005$ \\
\hline
\end{tabular}

4.3. Comparación de los resultados experimentales con los teóricos obtenidos mediante el modelo de Chen-Hashimoto.

A partir de la ecuación (4) se obtiene:

$$
S=\frac{\text { So }}{\left(1+\theta \mu_{\max } / K-1 / K\right)}
$$

que permite determinar los valores teóricos de concentración de sustrato biodegradable, $\mathrm{S}$, en función de los valores de $\mathrm{K}$ y $\mu_{\max }$ calculados previamente.

En la tabla IV se recogen los valores experimentales de $\mathrm{S}$, así como los valores teóricos obtenidos mediante la ecuación anterior. Según se observa existe un buen grado de ajuste entre ambos. Todos los valores teóricos de $S$, correspondientes a los distintos tiempos de retención ensayados en los dos intervalos de temperatura, se desvían menos del $5 \%$ de sus valores experimentales. En concreto, un $92,8 \%$ del total de los valores teóricos se desvian menos o igual de un $2 \%$ de sus correspondientes valores experimentales. Esto indica que el modelo ensayado predice el comportamiento de los reactores tanto en la fase mesofílica como en la termofílica y los parámetros cinéticos obtenidos representan la actividad de los microorganismos responsables del proceso continuo de digestión anaerobia del alpechín en los dos casos estudiados.
Tabla IV

Valores experimentales y teóricos de concentración de sustrato biodegradable, $\mathbf{S}(\mathrm{g} / \mathrm{l})$, en función del tiempo de retención hidráulico (días), para los dos casos estudiados

\begin{tabular}{cccccc}
\hline \multirow{2}{*}{$\begin{array}{c}\text { Tiempo } \\
\text { retención }\end{array}$} & \multicolumn{2}{c}{ Mesofílico } & & \multicolumn{2}{c}{ Termofílico } \\
\cline { 2 - 3 } \cline { 5 - 6 } & Experim. & Teórico & & Experim. & Teórico \\
\hline 40 & 1,05 & 1,07 & & 0,85 & 0,85 \\
35 & 1,30 & 1,27 & & 1,00 & 1,01 \\
30 & 1,55 & 1,56 & & 1,20 & 1,19 \\
25 & 2,05 & 2,04 & & 1,50 & 1,48 \\
20 & 2,90 & 2,92 & & 2,05 & 2,02 \\
15 & 5,15 & 5,17 & & 3,05 & 3,06 \\
10 & 21,25 & 22,31 & & 6,20 & 6,34 \\
\hline
\end{tabular}

4.4. Determinación de la velocidad volumétrica de utilización de sustrato

Una vez obtenidos los parámetros cinéticos, $\mu_{\max }$ y $\mathrm{K}$, el modelo de Chen-Hashimoto permite la determinación de la velocidad volumétrica de utilización de sustrato, $F$, para cada tiempo de retención, mediante la ecuación:

$$
F=\frac{S o}{\theta}\left[1-K\left(\mu_{\max } \theta-1+K\right)\right]
$$

En la figura 2 se representan estos valores en función del tiempo de retención hidráulico. Como se observa,

(g DQO/L/dia)

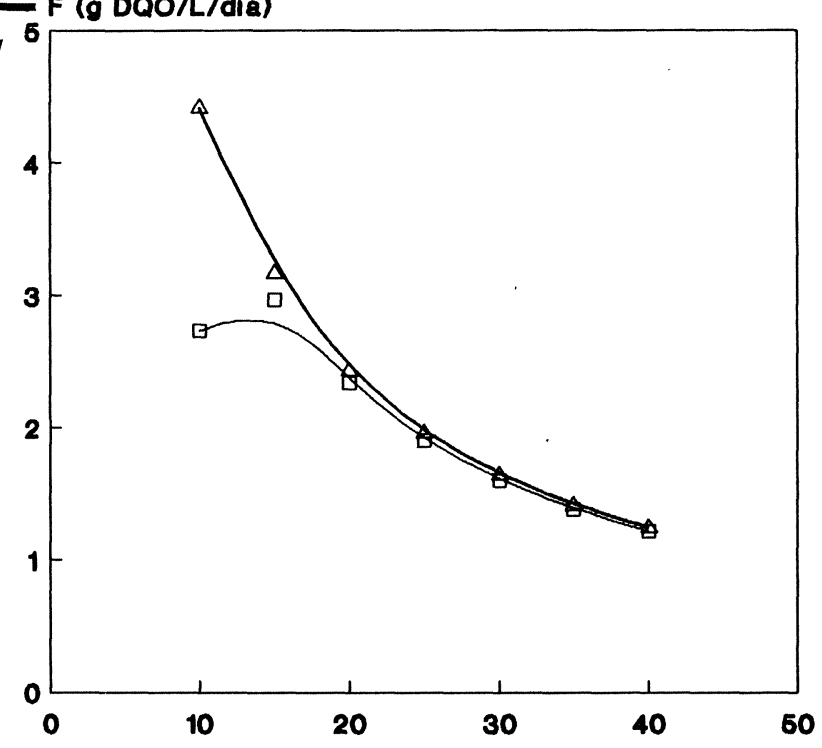

Tiempo de retención hidráulico (dias)

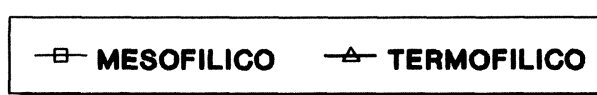

Figura 2

Evolución de la velocidad volumétrica de utilización de sustrato $\mathrm{F}$ (g DQO//dia) con el tiempo de retención hidráulico (dias). 
entre 15 y 40 días no se aprecia una diferencia significativamente importante entre los valores de $F$ correspondientes a los procesos mesofílico y termofílico, aunque los valores correspondientes al segundo son siempre ligeramente superiores a los del primero. En cambio para 10 días de tiempo de retención hidráulica, el valor de $F$ es un $62 \%$ mayor en el proceso termofílico $(4,4 \mathrm{~g} \mathrm{DQO} / \mathrm{l} /$ día) que en el mesofílico (2,7 g DQO///día). Asimismo, el porcentaje medio de eliminación de materia orgánica para tiempos de retención entre 15 y 40 dias, es un $95,3 \%$ y un $96,8 \%$ en los procesos mesofílico y termofílico, respectivamente. En cambio, para un tiempo de 10 días, los porcentajes de sustrato eliminado presentan los valores del $57,2 \%$ y $87,7 \%$, respectivamente.

Por otra parte, el modelo permite la determinación de la velocidad máxima volumétrica de utilización de sustrato y del tiempo de retención en el que ésta se alcanza, mediante las expresiones:

$$
\begin{aligned}
& F_{\max }= \frac{(1-R) \mu_{\max } \text { So }}{\left(1+K^{1 / 2}\right)^{2}} \\
& \theta=\left(1+K^{1 / 2}\right) / \mu_{\max }
\end{aligned}
$$

siendo $R$ la fracción en tanto por uno de materia orgánica no biodegradable.

La aplicación de estas ecuaciones a los datos obtenidos para los procesos mesofílico y termofílico da lugar a

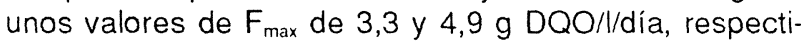
vamente, para tiempos de retención de 11,6 y 7,7 días.

Por tanto, se comprueba que el proceso termofílico da lugar a un valor superior de la velocidad máxima volumétrica de utilización de sustrato en relación al mesofílico, y que esta tiene lugar para un tiempo de retención más pequeño. Todo ello da idea de la idoneidad del proceso termofílico cuando el reactor trabaja con tiempos de retención hidráulica inferiores a 15 días.

\subsection{Determinación del coeficiente de producción} de metano

A partir de los datos de producción de metano para cada tiempo de retención (tabla II), se han calculado los valores del parámetro $B$, definido como: volumen de metano en mililitros por gramo de DQO añadido.

En la figura 3 se representan los valores de este parámetro en función del tiempo de retención. Se observa que entre 20 y 40 días el valor de este coeficiente se mantiene prácticamente constante, presentando un valor medio más elevado en el proceso anaerobio termofílico (338 $\left.\mathrm{ml} \mathrm{CH}_{4} / \mathrm{g} \mathrm{DQO}\right)$ que en el mesofílico $\left(286 \mathrm{ml} \mathrm{CH}_{4} / \mathrm{g}\right.$ DQO). Por otra parte, para tiempos de retención inferiores a 20 días se produce un descenso que resulta muy suave en el proceso termofílico, y muy acusado, por el contrario, para el proceso mesofílico, lo que demuestra la menor estabilidad de este último en relación con el primero, al aumentar el caudal de alimentación en los reactores. Este brusco descenso en la producción de metano del proceso mesofílico va acompañado de un aumento considerable en la acidez volátil del sistema, cuyos valo-
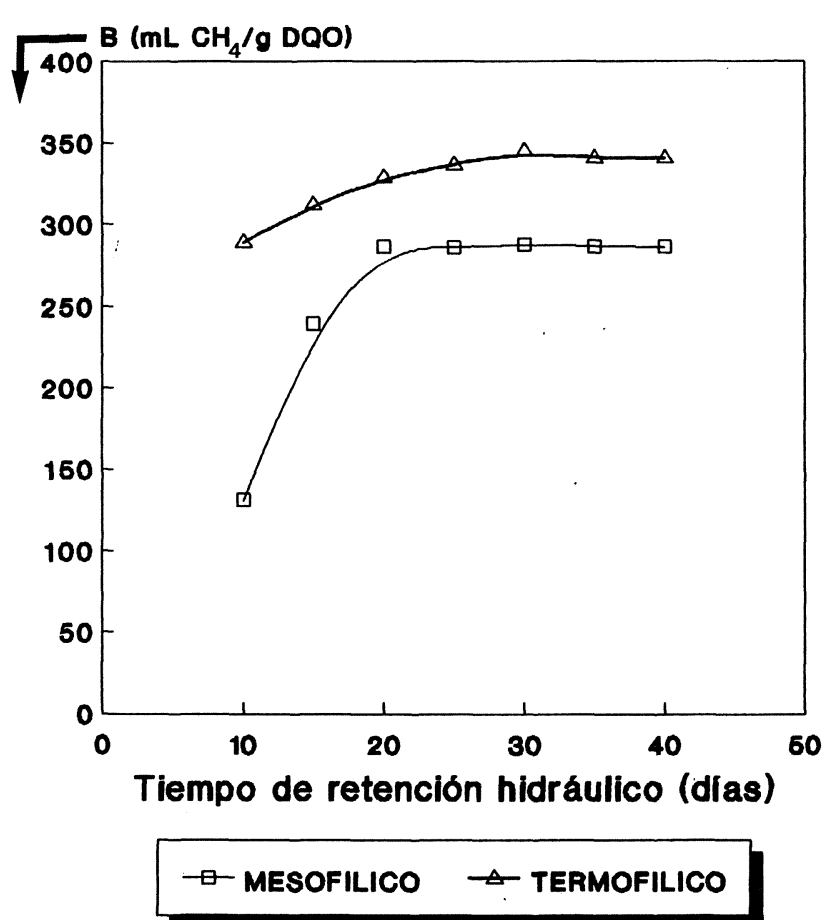

Figura 3

Evolución del coeficiente de producción de metano $\mathrm{B}\left(\mathrm{ml} \mathrm{CH}_{4} / \mathrm{g} \mathrm{DQO}\right)$ con el tiempo de retención hidráulico (días).

res pasan de una media de 250 ppm, expresado en ácido acético, para valores del tiempo de retención entre 20 y 40 días, a 1900 ppm para un tiempo de 10 días, lo que provoca un descenso en el pH desde 7,2 a 6,0. En cambio, en el proceso termofílico la acidez sólo aumenta de 200 a 375 ppm al pasar de 40 a 10 días de tiempo de retención, manteniéndose el pH entre 7,3 y 7,0 en este intervalo de tiempo.

\section{CONCLUSIONES}

1. Se ha comprobado que el modelo de utilización de sustrato propuesto por Chen-Hashimoto es aplicable al proceso continuo de digestión anaerobia del alpechín, tanto en la fase mesofílica como en la termofílica. Los parámetros cinéticos obtenidos predicen la concentración de sustrato en el efluente con un error igual o inferior al $2 \%$ en más del $92 \%$ de los casos estudiados.

2. Los parámetros cinéticos $\mu_{\max }$ y $\mathrm{K}$ son un $55 \%$ y un $34 \%$ mayores, respectivamente, en el proceso termofílico que en el mesofílico, al producirse un aumento en la cinética de las transformaciones celulares con la temperatura del sistema.

3. El porcentaje medio de eliminación de materia orgánica es superior al $95 \%$ en ambos casos, para tiempos de retención comprendidos entre 15 y 40 días; $\sin$ embargo para tiempos de 10 dias, los porcentajes de degra- 
dación disminuyen hasta un $87 \%$ y $57 \%$ para los procesos termofílico y mesofílico respectivamente.

4. El coeficiente de producción de metano es siempre superior en el proceso termofílico, y no disminuye bruscamente al disminuir el tiempo de retención como sucede en el mesofílico.

\section{AGRADECIMIENTOS}

Queremos hacer constar nuestro agradecimiento al Departamento de Postgrado y Especialización del CSIC y a la Dirección General de Universidades e Investigación de la Consejería de Educación y Ciencia de la Junta de Andalucía por su apoyo económico para la realización de estos estudios; y a D. ${ }^{a}$ Carmen Sánchez por su eficaz ayuda y colaboración.

\section{BIBLIOGRAFIA}

Benefield, D. and Randall, C.W. (1987).- "Design procedure for a contact stabilization activated sludge process".- J. Water Poll. Control Fed. $49(5), 869-872$

Borja, R., Martín, A. y Fiestas, J.A. (1991).- "Influencia de la concentración de sustrato sobre la cinética del proceso de biometanización del alpechín, en biorreactores de lecho fluidizado con microorganismos inmovilizados".- Grasas y Aceites 41, 363-370.

Borja, R., Martín, A., Olias, J.M., Fiestas, J.A. and Durán, M.M. (1992)."Behaviour of sepiolite and bentonite as immobilization supports in the anaerobic degradation of phenolic compounds present in olive mill wastewater".- Inquinamento 3, 114-117.

Buhr, H.O. (1977).- "The thermophilic anaerobic digestion process".Water Research 11, 129-143.

Chen, M. (1983).- "Adaptation of mesophilic anaerobic sewage fermentor populations to thermophilic temperatures".- App. Environmental Microbiology 45 (4), 1271-76.

Demharter, W. and Pfeiffer, W. (1988).- "Sewage sludge digestion and desinfection. A comparison of mesophilic and thermophilic proces- ses".- Fifth Internatıonal Symposium on Anaerobic Digestion, Bologna, Italy, 699-702.

Dhavises, G., Sriprasertsak, P., Tanaka, T., Taniguchi, M. and Oi, S. (1985).- "Mesophilic and thermophilic methane fermentation of agrowastes and grasses".- J. Ferment. Technol. 63 (1), 45-49.

Hashimoto, A.G. (1983).- "Conversion of straw-manure mixtures to methane at mesophilic and thermophilic temperatures".- Biotecnology and Bioengineering XXV, 185-200.

Kandier, O. (1983) - "Efficiency and stability of methane fermentation of wastes at mesophilic and thermophilic temperatures".- CHEMTECH Symp. on: Recent Advances in Biotechnology 18 (11), 57-65.

Lema, J.M.; Soto, M.; Méndez, R. and Blázquez, R. (1988).- "Comparison of mesophilic and thermophilic anaerobic filters treating very high saline wastewaters".- Fifth International Symposium on Anaerobic Digestion, Bologna, Italy, 547-549.

Marchaim, U. (1988).- "Thermophilic anaerobic digestion system for slaughter-house wastes: an economical and ecological solution".Fifth International Symposium on Anaerobic Digestion, Bologna, Italy, 555-556.

Monod. J. (1949).- "The growth of bacterial cultures".- Ann. Rev. Microbiol. 3, 371-6.

Pfeffer, J.T. (1984).- "Temperature effects on anaerobic fermentation of domestic refuse".- Biotechnol. Bioeng. 16 (6), 771-87.

Sales, D. Romero, LI: Valcárcel M.J. Pérez, L and Martínez de la Ossa, E. (1988).- "Thermophilic and mesophilic anaerobic digestion of wine-distillery wastewaters".- Fifth International Symposium on Anaerobic Digestion, Bologna, Italy, 575-8.

Sorlini, C. Andreoni, V.; Ferrari, A : Ranalli, G. (1986).- "The influence of some phenolic acids present in oil mill waters on microbic groups for the methanogenesis". - Proceedings del Simposium Internacional sobre la valoración de los subproductos del olivar, Sevilla, 81-8.

Sriprasertsak, P.: Dhavises, G. and Oi, S. (1985).- "Mesophilic and thermophilic methane fermentations of slop waste".- J. Ferment. Technol. 63 (6) 567-73.

Vicent. T. Paris, J.M. Lema, J.M. and Ibáñez, E. (1985)- - Thermophilic anaerobic treatment of an industrial wastewater: startup and stability studies".- Biotechnol. and Bioeng. 15, 599-609.

Whitmore. T.N.; Lazzari, M. and Lloyd, D. (1985).- "Comparative studies of methanogenesis in thermophilic and mesophilic anaerobic digesters using membranne inlet mass spectrometry".- Biotechnology Letters 7 (4), 283-288

(Recibido: Marzo 1992) 